\title{
CLASSIFICAÇÃO DA DEPENDÊNCIA DE CUIDADOS DE ENFERMAGEM DOS PACIENTES ACOMETIDOS POR ACIDENTE VASCULAR ENCEFÁLICO
}

\author{
Ana Rachel Cavalcante Araújo, Edilane Perote de Paula², Virna Ribeiro Feitosa Cestari³, Islene Victor Barbosa ${ }^{4}$, Zuíla Maria
} de Figueiredo Carvalho ${ }^{5}$

${ }^{1}$ Enfermeira. Especialista em Enfermagem em Centro de Terapia Intensiva. Universidade Estadual do Ceará. Fortaleza, CE, Brasil.

${ }^{2}$ Enfermeira. Universidade de Fortaleza. Fortaleza, CE, Brasil.

${ }^{3}$ Discente do curso de graduação em Enfermagem. Universidade de Fortaleza. Fortaleza, CE, Brasil.

${ }^{4}$ Enfermeira. Doutora em Enfermagem. Docente do Departamento de Enfermagem da Universidade de Fortaleza. Fortaleza, CE, Brasil.

${ }^{5}$ Enfermeira. Pós-doutora em Enfermagem. Docente do Departamento de Enfermagem da Universidade Federal do Ceará. Fortaleza, CE, Brasil.

RESUMO: Objetivou-se caracterizar os pacientes acometidos por Acidente Vascular Encefálico quanto aos aspectos sociodemográficos e classificar esses indivíduos segundo o grau de dependência dos cuidados de enfermagem. Estudo transversal, com abordagem quantitativa, desenvolvido com 100 pacientes, em um hospital público, situado em Fortaleza, estado do Ceará. Os dados foram coletados no período de julho a setembro de 2012, por meio de um instrumento contendo informações referentes aos dados sociodemográficos e aplicação da Escala de Classificação de Pacientes. Os dados categóricos foram analisados por meio de distribuição de frequência absoluta e relativa. Verificou-se que 58\% dos pacientes exigiam cuidados intermediários; $22 \%$, cuidados semi-intensivos; $18 \%$, cuidados mínimos e $2 \%$, intensivos. O uso do processo de categorização do paciente torna favorável o planejamento das ações de cuidados de enfermagem para coadunar esforços, no intuito de oferecer assistência de qualidade a esta clientela.

DESCRITORES: Acidente vascular cerebral; Enfermagem; Avaliação em enfermagem.

\section{NURSING CARE DEPENDENCY SCALE FOR PATIENTS AFFECTED BY CEREBROVASCULAR ACCIDENT}

\begin{abstract}
The present study aimed to characterize patientswho had a strokeregarding socio-demographic aspects and classify these individuals according to their degree of dependence on nursing care.Qualitative crosssectional study with 100 patients conducted in a public hospital, in Fortaleza, state of Ceará.Data were collected from July to September 2012 through an instrument containing socio-demographic information and the application of a Patient Rating Scale.Categorical data were analyzed using absolute and relative distribution frequency. It was found that $58 \%$ of the patients required intermediate care; $22 \%$, semi-intensive care; $18 \%$, minimal care an $2 \%$, intensive care. The use of a patient's rating process favors the planning of nursing care, joining efforts to provide quality care to this clientele.
\end{abstract}

DESCRIPTORS: Cerebrovascular accident; Nursing; Assessment in nursing.

\section{CLASIFICACIÓN DE LA DEPENDENCIA DE CUIDADOS DE ENFERMERÍA DE LOS PACIENTES ACOMETIDOS POR ACCIDENTE VASCULAR ENCEFÁLICO}

RESUMEN: Fue objetivo del estudio caracterizar los pacientes acometidos por Accidente Vascular Encefálico cuanto a los aspectos sociodemográficos y clasificar esos individuos según el grado de dependencia de los cuidados de enfermería. Estudio transversal, con abordaje cuantitativo, desarrollado con 100 pacientes, en un hospital público, ubicado en Fortaleza, estado de Ceará. Los datos fueron obtenidos en el periodo de julio a septiembre de 2012, por medio de un instrumento con informaciones de los datos sociodemográficos y aplicación de la Escala de Clasificación de Pacientes. Los datos categóricos fueron analizados por medio de distribución de frecuencia absoluta y relativa. Se verificó que $58 \%$ de los pacientes exigían cuidados intermediarios; $22 \%$, cuidados semintensivos; $18 \%$, cuidados mínimos y $2 \%$, intensivos. El uso del proceso de categorización del paciente vuelve el planeamiento de las acciones de cuidados de enfermería favorable para coadunar esfuerzos, a fin de ofrecer asistencia de cualidad a esta clientela.

DESCRIPTORES: Accidente vascular cerebral; Enfermería; Evaluación en enfermería. 


\section{INTRODUÇÃO}

O Acidente Vascular Encefálico (AVE) é uma patologia causada pela perda repentina da função cerebral resultante do rompimento ou diminuição, total ou parcial, do suprimento sanguíneo ao encéfalo(1). É um conjunto de sintomas neurológicos focais ou globais, que duram mais que 24 horas, tendo início abrupto ou em forma de crise, caracterizada por resultar em grandes níveis de incapacidade e dependência funcional do paciente, estando associada à deterioração das capacidades físicas, cognitivas, emocionais e sociais do indivíduo ${ }^{(2)}$.

Em escala mundial, o AVE é a segunda causa de morte, predominantemente em adultos de meia-idade e idosos ${ }^{(3)}$. No Brasil, a mudança do perfil de morbimortalidade com as doenças crônicas não transmissíveis é evidente: em 2009, foram registradas 160.621 internações por doenças cerebrovasculares. A taxa de mortalidade foi de 51,8 a cada grupo de 100.000 habitantes $^{(4)}$.

Estudo mostra que aproximadamente $20 \%$ dos pacientes com AVE sobrevivem apenas um mês após sua ocorrência, outros $50 \%$ sobrevivem por mais tempo, mas apresentam deficiência, considerável e permanente, necessitando de cuidados. Os demais 30\% apresentam déficits neurológicos, adquirindo permanentemente certo grau de dependência ${ }^{(5)}$.

Diante dos diferentes níveis de incapacidades, a assistência de enfermagem ao paciente com AVE deve levar em conta as necessidades dos mesmos. Nesse contexto, o desenvolvimento de Sistemas de Classificação de Pacientes (SCP) surgiu da necessidade do aprimoramento do planejamento dos recursos humanos em instituições de saúde.

O SCP é um método que viabiliza o cuidado individualizado, com o intuito de melhorar os padrões de qualidade assistencial por permitir a identificação do perfil assistencial dos pacientes, subsidiar a realocação de recursos humanos e materiais, reorientar a dinâmica assistencial e determinar os custos da assistência de enfermagem ${ }^{(6)}$.

Em 1996, foi desenvolvido por Perroca(7) um instrumento para nortear a classificação de pacientes por tipo de cuidado, baseado nas necessidades individualizadas de cuidado de enfermagem. $O$ instrumento envolve a avaliação de 13 indicadores críticos do cuidado, que abrangem tanto a esfera biológica como a psicossocial do cuidado. São eles: Estado mental e Nível de consciência; Oxigenação; Sinais vitais; Nutrição e Hidratação; Motilidade; Locomoção; Cuidado corporal; Eliminações; Terapêutica; Educação para a saúde; Comportamento; Comunicação; e Integridade cutâneo-mucosa.

Diversos estudos que aplicaram esse sistema de classificação estão voltados para a investigação do grau de dependência em relação aos cuidados de enfermagem, em unidades de internação $^{(6-8)}$. No entanto, verificou-se a ausência de estudos realizados no Brasil avaliando o grau de dependência ou a gravidade de pacientes acometidos por AVE pela referida escala.

É imprescindível para os enfermeiros a incorporação de conhecimentos científicos atualizados para instrumentalizar sua prática clínica. O SCP na enfermagem diz respeito à necessidade de engajamento de todos os enfermeiros nesse processo, para que possam utilizá-los na sua prática profissional, nos setores gerais e especializados, beneficiando o paciente e o profissional em si. Pretende-se, portanto, contribuir e somar esforços para a melhoria da prática assistencial da enfermagem e incentivar a realização de novos estudos que envolvam a temática.

Frente às colocações citadas, propôs-se a presente investigação com os objetivos de caracterizar os pacientes acometidos por AVE quanto aos aspectos sociodemográficos e classificar esses indivíduos segundo o grau de dependência dos cuidados de enfermagem.

\section{MÉTODO}

Trata-se de um estudo transversal, observacional, descritivo, com abordagem quantitativa. A pesquisa foi realizada em um hospital conveniado ao Sistema Único de Saúde (SUS), situado em Fortaleza, estado do Ceará, Brasil. A escolha pela referida instituição deuse por esta possuir unidade de atendimento especializada em pacientes acometidos por AVE, sendo referência para o estado.

A população foi composta por 100 pacientes internados com o diagnóstico de AVE. Os critérios de inclusão foram: a) ter idade acima de 18 anos; b) ter o diagnóstico médico de $\mathrm{AVE} ; \mathrm{c}$ ) aceitar participar da pesquisa mediante a assinatura do Termo de Consentimento Livre e Esclarecido. Foram excluídos do estudo os pacientes com diagnóstico médico não estabelecido. A amostra foi selecionada por conveniência. 
Os dados foram coletados no período de julho a setembro de 2012. Foi utilizado inicialmente um formulário estruturado que continha questões acerca das variáveis sociodemográficas e, posteriormente, um instrumento de classificação criado e validado por Perroca(7) que pontua o grau de dependência dos pacientes em relação à assistência de enfermagem. $O$ instrumento em questão passou por uma atualização de seu conteúdo e renovação de sua estrutura, permitindo mensuração mais acurada da complexidade assistencial dos pacientes e dos recursos de enfermagem utilizados pelos $\operatorname{mesmos}^{(9)}$.

O instrumento utiliza 13 indicadores críticos de cuidado, graduados de um a cinco, apontando a intensidade crescente de complexidade do cuidado, de forma que o valor 1 corresponde ao nível menor de complexidade assistencial, e o valor 5, ao nível máximo de complexidade assistencial. Desta forma, o valor obtido individualmente, em cada um dos indicadores, é somado, e o valor total é obtido comparado com os intervalos de pontuações propostos, conduzindo, dessa forma, a uma classe ou categoria de cuidado a que esse paciente pertence: mínimos (13 a 26 pontos), intermediários (27 a 39 pontos), semi-intensivos ( 40 a 52 pontos) e intensivos ( 53 a 65 pontos).

Para a análise, os dados foram transcritos e tabulados em banco de dados do programa Excel Microsoft XP, versão 2010. Os dados categóricos foram apresentados com frequências absolutas e relativas, em tabelas e gráficos, e discutidos de acordo com a literatura pertinente ao tema.

Ressalta-se que a pesquisa foi aprovada pelo Comitê de Ética e Pesquisa da Universidade de Fortaleza (UNIFOR) sob o parecer $\mathrm{n}^{\circ}$ 03110303/11, em concordância com as determinações da Resolução $n^{\circ}$ 196/96 do Conselho Nacional de Saúde ${ }^{(10)}$.

\section{RESULTADOS}

Para caracterização da amostra foram investigados: sexo, faixa etária, naturalidade, situação conjugal, religião, renda familiar, ocupação, nível de escolaridade (em anos). Esses dados estão expostos na Tabela 1.

$\mathrm{Na}$ amostra estudada observou-se a equivalência entre os sexos, sendo 50 (50\%) indivíduos do sexo feminino e 50 (50\%) do masculino; a predominância de participantes com idade acima de 60 anos, 59 (59\%); naturais do interior, 75 (75\%); e com companheiros, 60 (60\%).
Tabela 1 - Distribuição dos pacientes acometidos por AVE de acordo com as variáveis sociodemográficas. Fortaleza, CE, Brasil, 2012

\begin{tabular}{|c|c|c|}
\hline Variáveis & $\mathbf{N}$ & $\%$ \\
\hline \multicolumn{3}{|l|}{ Sexo } \\
\hline Feminino & 50 & 50 \\
\hline Masculino & 50 & 50 \\
\hline \multicolumn{3}{|l|}{ Faixa etária } \\
\hline$>60$ anos & 59 & 59 \\
\hline$<60$ anos & 41 & 41 \\
\hline \multicolumn{3}{|l|}{ Naturalidade } \\
\hline Capital & 18 & 18 \\
\hline Interior & 75 & 75 \\
\hline Outros & 7 & 7 \\
\hline \multicolumn{3}{|l|}{ Situação conjugal } \\
\hline Com companheiro & 60 & 60 \\
\hline Sem companheiro & 40 & 40 \\
\hline \multicolumn{3}{|l|}{ Religião } \\
\hline Católico & 78 & 78 \\
\hline Evangélico & 17 & 17 \\
\hline Testemunha de Jeová & 1 & 1 \\
\hline Sem religião & 4 & 4 \\
\hline \multicolumn{3}{|l|}{ Renda familiar } \\
\hline$<01$ salário mínimo & 6 & 6 \\
\hline 01 salário mínimo & 52 & 52 \\
\hline 02 a 04 salários mínimos & 36 & 36 \\
\hline >04 salários mínimos & 6 & 6 \\
\hline \multicolumn{3}{|l|}{ Ocupação } \\
\hline Aposentado & 51 & 51 \\
\hline Exerce atividade remunerada & 49 & 49 \\
\hline \multicolumn{3}{|l|}{ Nível de escolaridade (em anos) } \\
\hline De 01 a 03 anos & 31 & 31 \\
\hline De 04 a 07 anos & 30 & 30 \\
\hline De 08 a 11 anos & 22 & 22 \\
\hline 12 ou mais anos & 17 & 17 \\
\hline
\end{tabular}

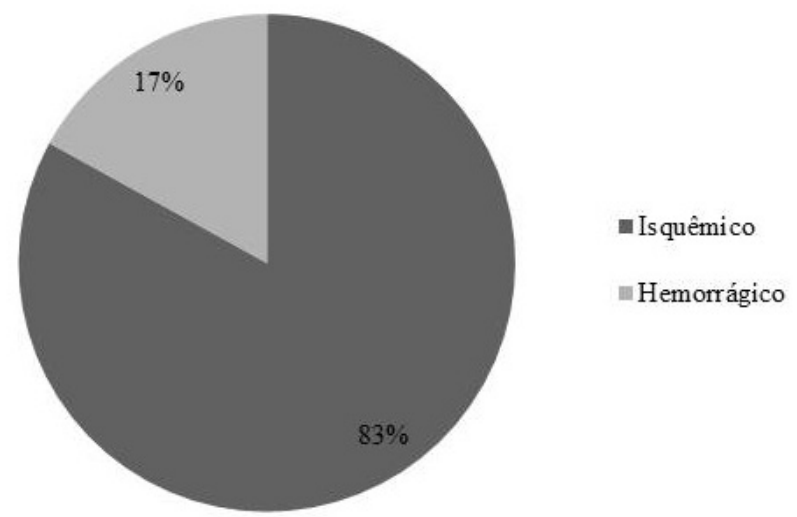

Figura 1 - Distribuição dos pacientes segundo tipo de AVE. Fortaleza, 2012 


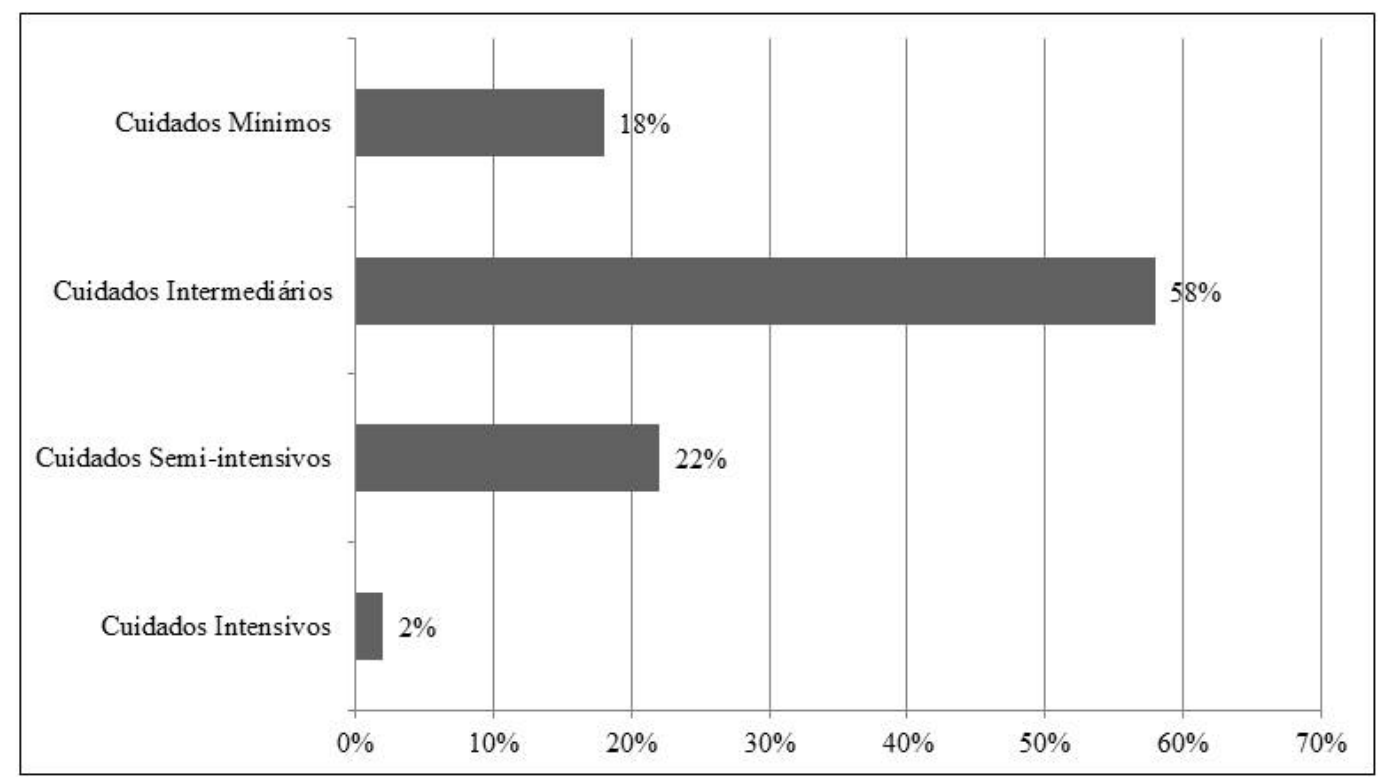

Figura 2 - Distribuição da classificação de cuidados de enfermagem dos pacientes acometidos AVE. Fortaleza, 2012

No tocante à religião, $78(78 \%)$ eram católicos, seguido de 17 (17\%) evangélicos. O nível de escolaridade que predominou foi de 01 a 03 anos de estudo, em 31 (31\%) participantes, seguido de 04 a 07 anos de estudo, em 30 (30\%).

A renda familiar evidenciou que 52 (52\%) participantes deste estudo possuíam uma renda de um salário mínimo vigente no período do estudo. Em análise à ocupação da amostra, obtevese uma predominância de pacientes aposentados, 51 (51\%). Em análise ao tipo de AVE, constatou-se a quase totalidade de ocorrência do isquêmico (AVEi) (Figura 1).

A Figura 2 apresenta a distribuição dos pacientes quanto ao grau de dependência, de acordo com o sistema de classificação. Observase que $58(58 \%)$ dos pacientes foram classificados em cuidados intermediários.

\section{DISCUSSÃO}

Caracterizou-se, neste estudo, o perfil dos pacientes acometidos por AVE e a sua classificação, segundo o grau de dependência dos cuidados de enfermagem proposto, e houve equivalência entre o quantitativo de pacientes segundo o sexo masculino e feminino. Os achados de outros estudos realizados com essa clientela evidenciam que a ocorrência do AVE não tem sido associada ao sexo ${ }^{(11-13)}$.

Em estudo(11) realizado com 180 pacientes que apresentaram o diagnóstico médico de AVE, em Fortaleza, estado do Ceará, verificou-se que este acometeu tanto homens $(48,9 \%)$ quanto mulheres $(51,1 \%)$, sem diferença estatística entre eles $(p<0,134)$. Outra pesquisa ${ }^{(12)}$ realizada com 47 pacientes com AVE, em Minas Gerais, verificouse predominância discretamente maior na população masculina $(54,7 \%)$. Esses dados estão de acordo com uma pesquisa realizada na Itália ${ }^{(13)}$ com 362 indivíduos, que evidenciou pequena prevalência da doença no sexo masculino $(57,8 \%)$.

Os dados indicaram a predominância de pacientes com idade acima dos 60 anos. A associação de faixas etárias mais elevadas com a ocorrência de doenças cerebrovasculares já é conhecida e justificada, principalmente, pela predisposição biológica para estas doenças inerente ao avanço da idade, independentemente de outros fatores de risco.

Comparandonossos dados comoutroestudo ${ }^{(14)}$, em que se buscou investigar as hospitalizações de adultos decorrentes de AVE isquêmico ocorridas no Brasil, entre os anos de 2006 a 2007, verificouse que, de 16.879 internações, a idade média dos indivíduos foi de 64,7 anos (DP=15,7 anos).

A idade é o principal fator de risco não modificável para AVE, o que reforça a necessidade de medidas urgentes na prevenção e controle destes, a serem realizadas pela atenção básica ${ }^{(14-15)}$. Estratégias de educação em saúde e rastreamento de doenças que possam causar AVE são essenciais para a promoção da redução das vulnerabilidades da população em geral.

Os resultados da pesquisa indicam que a maioria dos participantes é originária do interior do estado. Pode-se considerar que 
esses participantes poderiam apresentar alguma doença crônica, como hipertensão arterial sistêmica, diabetes, cardiopatias ou hábitos de vida não saudáveis como tabagismo e etilismo, que são fatores de risco para as doenças cerebrovasculares.

Observou-se que a maioria dos pacientes tinha um companheiro. Dependendo da gravidade do comprometimento neurológico, o AVE acarreta à pessoa deficiências neurológicas e incapacidades residuais significativas, tanto na função motora, como cognitiva. Com a perda da autonomia, há necessidade de auxílio de terceiros para a realização das ações cotidianas básicas ${ }^{(5)}$. Dessa forma, pode-se inferir que a situação conjugal dos participantes do estudo é um fator positivo, uma vez que os mesmos vivem o desapontamento e a incerteza sobre o futuro e perdem a independência, principalmente para as atividades de vida diária, necessitando de acompanhamento para realizá-las, bem como no enfrentamento das dificuldades.

Estudo $^{(16)}$ sobre o perfil de cuidadores de pessoas acometidas pelo AVE, evidenciou que, de 83 pacientes, $70 \%$ eram cuidados pelo cônjuge. Esses dados corroboram com os achados de uma pesquisa ${ }^{(17)}$ descritiva que mostrou que entre 242 cuidadores, 78,6\% eram mulheres e esposas. Dados de uma pesquisa ${ }^{(18)}$ realizada em Fortaleza, estado do Ceará, com 52 cuidadores familiares destacam os filhos $(63,5 \%)$ como os principais cuidadores. Independente da relação familiar, constata-se que, diante das incapacidades advindas pela doença, é essencial a presença de um companheiro ou pelo menos um cuidador capaz de ajudar os clientes nas suas atividades básicas.

Verificou-se o predomínio da religião católica. A influência da religiosidade tem demonstrado um grande impacto sobre a saúde física, definindo-se como possível fator de prevenção ao desenvolvimento de doenças na população e eventual redução de óbito ou impacto de diversas doenças. As evidências têm se direcionado de forma mais firme e consistente para o cenário de prevenção. Estudos tentando avaliar qual a relação entre redução de mortalidade e prática religiosas têm enfatizado o possível incentivo que essas práticas oferecem a hábitos de vida saudáveis, suporte social, menores taxas de estresse e depressão(19). Percebe-se, portanto, que a religião pode funcionar como uma válvula de escape, como apoio para superar uma nova condição de vida.
A maioria dos participantes deste estudo tinha como renda familiar de um a quatro salários mínimos. Esse dado é preocupante, visto que caracteriza menos disponibilidade de recursos para a qualidade alimentar, educacional, de saúde e de recursos gerais ${ }^{(20)}$. A classe econômica destaca-se como fator de risco associado à ocorrência da doença e à percepção regular/ruim de saúde. Esse dado está de acordo com uma pesquisa transversal(21), realizada em Maringá, estado do Paraná, com 1.232 indivíduos com AVE. Os autores verificaram que as classes econômicas mais predominantes entre os indivíduos eram a D (de dois a quatro salários mínimos) e C (quatro a dez salários mínimos). Nesses indivíduos, a autopercepção de saúde foi negativa, quando comparada com as classes A e B $(p<0,019)$.

Os dados evidenciaram a baixa escolaridade da amostra, em até três anos de estudo. O baixo nível de escolaridade pode colaborar para o aparecimento da doença, pois esse fato, associado aos fatores econômicos e culturais, pode dificultar a conscientização para as necessidades de cuidado com a saúde ao longo da vida, tratamento e manutenção de estilo de vida saudável. Em estudo descritivo(22) realizado em Fortaleza, estado do Ceará, a baixa escolaridade esteve fortemente associada a maior prevalência de doenças crônicas, que podem predispor à ocorrência do AVE.

Verificou-se que houve equivalência entre indivíduos aposentados e aqueles que exerciam alguma atividade remunerada. As sequelas oriundas do AVE geram impacto econômico, social e familiar, por causar, à maioria dos acometidos, prejuízo na capacidade funcional. Desta forma, esta patologia é responsável por grande parte das aposentadorias por invalidez $z^{(23)}$.

A amostra nesta pesquisa apresentou, majoritariamente, AVEi com $83 \%$ dos casos. Dados evidenciam que este corresponde aos casos mais comuns, sendo caracterizado pela interrupção do fluxo sanguíneo para o cérebro por um coágulo (trombo) ou por interrupção de atividade elétrica ${ }^{(3-5)}$. Nossos dados são semelhantes aos encontrados em estudo realizado com 74 pacientes portugueses acometidos pela doença, entre os quais $78,6 \%$ tiveram $\operatorname{AVEi}(24)$. $\mathrm{Em}$ pesquisa etnográfica instrumental, que teve como população 10 idosos com primeiro episódio de AVE, $90 \%$ destes apresentaram o tipo isquêmico ${ }^{(25)}$.

No presente estudo, os dados indicam que a classificação do grau de dependência 
estava entre os cuidados intermediários (58\%) e semi-intensivos (22\%). O conhecimento do perfil assistencial dos pacientes representa um fator que pode subsidiar o planejamento e a implementação de programas assistenciais que melhor atendam às necessidades dessa clientela, auxiliando na distribuição diária e na capacitação dos recursos humanos de enfermagem para o atendimento de cada grupo de pacientes ${ }^{(6)}$.

$\mathrm{Na}$ prática, quando um instrumento permite ao enfermeiro classificar os pacientes, conforme a assistência requerida, em mínima, intermediária, semi-intensiva e intensiva, proporciona que se tenha um quadro de pessoal quali-quantitativamente adequado para o desenvolvimento do cuidado, que deve ser prestado com eficácia e segurança(8). Entre os participantes deste estudo, houve maior prevalência de pacientes classificados em cuidados intermediários, destinados a pacientes estáveis, porém com parcial dependência de cuidados de enfermagem ${ }^{(9)}$. Os indicadores que mais contribuíram para o aumento do grau de dependência foram: locomoção, cuidado corporal, terapêutica e eliminações.

As sequelas mais comuns oriundas do AVE incluem a alteração na mobilidade física, responsável pelo aumento no risco de quedas, disfagia, afasia, desordens perceptuais cognitivas, depressão e outras perturbações emocionais e comportamentais ${ }^{(12-13)}$. Percebe-se que, com a doença, surgem diferentes níveis de incapacidade, aumentando consideravelmente o grau de dependência de cuidados de enfermagem.

Os achado desta pesquisa corroboram com estudos publicados na literatura nacional que abordam as incapacidade físicas decorrentes do $\operatorname{AVE}(1,25)$. Em estudo retrospectivo ${ }^{(25)}$ realizado em uma enfermaria de reabilitação neurológica situada em Brasília, Distrito Federal, observouse que, de 72 pacientes que apresentavam lesão cerebral decorrente de AVE, 69\% apresentaram hemiparesia; $18 \%$, hemiplegia; $10 \%$, tetraplegia; e $1 \%$, outros tipos de comprometimento motor. A cadeira de rodas foi a forma de auxílio mais utilizada por $50 \%$ dos pacientes. O mesmo estudo identificou, ainda, alterações de memória e comunicação em $56 \%$ e $57 \%$ dos indivíduos acometidos pelo AVE. Quanto às alterações intestinais, os autores evidenciaram a ocorrência da incontinência anal e constipação intestinal em $18 \%$ e $29 \%$ da população estudada.

A disfunção intestinal é uma condição comum após o AVE e está associada às desordens neurológicas, que levam a distúrbios nos esfíncteres vesicais e anorretal. Em pacientes hemiplégicos, essa disfunção também é observada e está relacionada à dependência, modificação do regime alimentar e condições de defecação ${ }^{(2)}$.

Os cuidados higiênicos envolvem o banho e a higiene oral. A higiene oral é uma prática utilizada para limpar a cavidade bucal, em especial os dentes e a língua, e inclui a escovação dos dentes, limpeza da língua, uso do fio dental, além de cuidados com dentaduras e pontes móveis ${ }^{(12)}$. Os cuidados básicos para a higiene corporal são essenciais para a manutenção da vida, portanto, devem fazer parte das ações do enfermeiro.

Evidenciou-se, ainda, a necessidade de maiores cuidados com relação à terapêutica dos pacientes deste estudo. Por conta do comprometimento cognitivo, de memória e de comunicação, o planejamento, a efetivação do autocuidados e a adesão à terapia medicamentosa são um desafio para o paciente. Torna-se essencial, nesse contexto, a inclusão da família na sua recuperação $\mathrm{O}^{(1)}$.

Quantidade representativa de pacientes deste estudo estava com o grau de necessidade de cuidados semi-intensivos, definidos como cuidados destinados a pacientes recuperáveis ou crônicos, estáveis clinicamente, mas com total dependência das ações de enfermagem ${ }^{(9)}$. Esse dado é preocupante, visto que a unidade neurológica comporta pacientes passíveis de instabilidade das funções vitais, requerendo assistência de enfermagem e médica permanente e especializada.

A permanência desses pacientes nessas unidades possibilita refletir se isto ocorre devido à insuficiência de leitos na Unidade de Terapia Intensiva para atender a demanda, ou pela falta de uma avaliação sistemática em relação à complexidade dos pacientes, comprometendo sua alocação em consonância com o perfil assistencial.

Em uma revisão integrativa ${ }^{(26)}$, que buscou identificar as intervenções de enfermagem aos pacientes hospitalizados por AVE, evidenciouse a necessidade de intervenções assistenciais e gerenciais. Os autores citaram, entre as intervenções assistenciais: reabilitação motora e funcional; administração de medicamentos; monitoramento das funções fisiológicas; planejamento para alta do paciente; cuidado emocional; cuidados para a prevenção de complicações e traumas; avaliação para o uso 
da terapia trombolítica; avaliação neurológica; cateterismo urinário; cuidados relacionados às atividades de autocuidado; posicionamento correto do paciente no leito; cuidados para prevenção da aspiração, entre outros.

Com relação às intervenções gerenciais, destacaram: coordenação do cuidado; organização, avaliação e coordenação do tratamento que será necessário no domicílio; e transferência do paciente para outros setores do hospital. O enfermeiro, dentro da equipe multidisciplinar, deve avaliar as necessidades do paciente e seus familiares, prover os recursos necessários para implementação dos cuidados prestados ao paciente e facilitar as transições no atendimento, buscando resultados que evidenciam cuidado de qualidade ${ }^{(2)}$.

O uso do SCPé uma importante ferramenta para monitoramento contínuo da carga de trabalho da equipe de enfermagem, por meio da identificação da complexidade assistencial do paciente ${ }^{(7)}$. Estudo $^{(27)}$ que objetivou avaliar a distribuição de pessoal de enfermagem em uma unidade de internação, evidenciou que nesta havia menor proporção de profissionais de enfermagem/ leito $(0,94)$. Os autores verificaram, também, o maior percentual de pacientes nas categorias de cuidados semi-intensivos (12,3\%) e intensivos $(2 \%)$ e o menor número de horas dos enfermeiros $(0,5)$ em relação à assistência de enfermagem. Esses valores mostram que a previsão e a distribuição de pessoal de enfermagem realizadas de maneira empírica, com base apenas na experiência clínica, pode gerar cálculos inapropriados à realidade organizacional, sobrecarregando a equipe de enfermagem.

\section{CONCLUSÃO}

A realização deste estudo contribuiu para o conhecimento do perfil dos clientes internados com AVE e, através da aplicação de um instrumento de classificação de pacientes, possibilitou caracterizá-los segundo o grau de dependência em relação ao cuidado de enfermagem.

Entre os indivíduos acometidos por AVE, neste estudo, não houve divergência quanto ao sexo, a faixa etária predominante foi acima dos 60 anos, oriundos do interior do estado, com companheiros, católicos, com renda familiar de um a quatro salários mínimos, baixa escolaridade e aposentados. Quanto ao tipo de AVE de maior ocorrência entre os participantes, destacou-se o isquêmico. Com relação à classificação do grau de dependência dos cuidados de enfermagem, obtidos através da aplicação da escala de Perroca, verificou-se que os pacientes tinham grau de dependência entre cuidados intermediários e semi-intensivos.

De acordo com o exposto, fica clara a importância de um processo de categorização do paciente em relação aos cuidados de enfermagem, além de um instrumento gerencial que possibilite aos enfermeiros avaliar, planejar e distribuir o quantitativo necessário de recursos humanos em instituições hospitalares.

É imprescindível para os enfermeiros a incorporação de conhecimentos científicos atualizados para instrumentalizar sua prática clínica. Com a utilização do SCP torna-se favorável o planejamento das ações de cuidados de enfermagem para coadunar esforços no intuito de oferecer assistência de enfermagem de qualidade para essa clientela.

\section{REFERÊNCIAS}

1. Lessmann JC, Conto F, Ramos G, Borenstein MS, Meirelles BHS. Atuação da enfermagem noautocuidado e reabilitação de pacientes que sofreram acidente vascular encefálico. Rev Bras Enferm. 2011;64(1):198202.

2. Bianchini SM, Galvão CM, Arcuri EAM. Cuidado e enfermagem ao paciente com acidentes vascular encefálico: revisão integrativa. Online Braz J Nurs. 2010; 9(2).

3. Vangen-Lonne AM, Wilsgaard $\mathrm{T}$, Johnsen $\mathrm{SH}$, Carlsson M, Mathiesen EB. Time trends in incidence and case fatality of ischemic stroke: The Thomso Study 1977 - 2010. Stroke. 2015;46(5):1173-9.

4. Almeida SRM. Análise epidemiológica do acidente vascular cerebral no Brasil. Rev Neurocienc. 2012;20(4):481-2.

5. Oliveira ARS, Araújo TL, Costa AGS, Morais HCC, Silva VM, Lopes MVO. Avaliação de pacientes com acidente vascular cerebral acompanhados por programas de assistência domiciliária. Rev. Esc. Enferm. USP. 2013;47(5):1147-53.

6. Lima LB, Borges D, Costa S, Rabelo ER. Classificação de pacientes segundo o grau de dependência dos cuidados de enfermagem e a gravidade em unidade de recuperação pós-anestésica. Rev. Latino-Am. Enferm. 2010;18(5):881-7

7. Perroca MG. Sistema de Classificação de pacientes: construção e validação de um instrumento [dissertação]. São Paulo (SP): Universidade de São Paulo; 1996. 
8. Zimmermann LP, Magnago TSBS, Urbanetto JS, Greco PBT, Viero NC, Vieira TG, et al. Avaliação do grau de dependência de cuidados de enfermagem dos pacientes internados em pronto-socorro. Rev Enferm UFSM. 2011;1(2):153-63.

9. Perroca MG. Desenvolvimento e validação de conteúdo da nova versão de um instrumento para classificação de pacientes. Rev. Latino-Am. Enferm. 2011;19(1):58-66.

10. Ministério da Saúde (BR). Conselho Nacional de Saúde. Diretrizes e normas regulamentadoras de pesquisas envolvendo seres humanos. Resolução n. 196, de 10 de outubro de 1996. Brasília; 1996.

11. Cavalcante TF, Moreira RP, Araújo TL, Lopes MVO. Fatores demográficos e indicadores de risco de acidente vascular encefálico: comparação entre moradores do município de Fortaleza e o perfil nacional. Rev. LatinoAm.Enferm. 2010; 18(4):703-8.

12. Scalzo PL, Souza ES, Moreira AGO, Vieira DAF. Qualidade de vida em pacientes com acidente vascular cerebral: clínica de fisioterapia Puc Minas Betim. Rev Neurocienc. 2010;18(2):139-44.

13.Ciccone A, Valvassori L, Nichelatti M, Sgoifo A, Ponzio M, Sterzi R, et al. Endovascular treatment for acute ischemic stroke. N Engl J Med. 2013;368(10):90413.

14. Rolim CLRC, Martins M. Qualidade do cuidado ao acidente vascular cerebral isquêmico no SUS. Cad. Saúde Pública. 2011;27(11):2106-16.

15. Zhou Q, Tang AK, Ombrello AK, Zavialov AV, Toro C, Zavialov AV, et al. Early-onset stroke and vasculopathy associated with mutations in ADA2. $\mathrm{N}$ Engl J Med. 370(10):911-20.

16. Lima ML, Santos JLF, Sawada NO, Lima LAP. Qualidade de vida de indivíduos com acidente vascular encefálico e de seus cuidadores de um município do Triângulo Mineiro. Rev Bras epidemiol. 2014;17(2):45364

17. McLennon SM, Bakas T, Jessup NM, Habermann B, Weaver MT. Task difficult and life changes among stroke family caregivers: relationship to depressive symptoms. Arch Phys Med Rehabil. 2014;95(12):248490.

18. Vieira CPB, Fialho AVM. Perfil de cuidadores familiares de idosos com acidente vascular cerebral isquêmico. Rev Rene. 2010;11(2):161-9.

19.Santos CCE, Gomes AMT, Oliveira DC, Pontes APM, Santos EI, Costa CPM. Diálogos entre espiritualidade e enfermagem: uma revisão integrativa da literatura. Cogitare enferm. 2013;18(2):372-8.
20. Moreira RP, Araújo TL, Cavalcante TF, Oliveira ARS, Holanda GF, Morais HCG, et al. Cuidador de cliente com acidente vascular encefálico: associação com diagnóstico e enfermagem. Rev Eletr Enferm. 2010; 12(3):425-30.

21. Arruda GO, Santos AL, Teston EF, Cecílio HPM, Radovanovic CAT, Marcon SS. Associação entre autopercepção de saúde e características sociodemográficas com doenças cardiovasculares em indivíduos adultos. Rev. Esc. Enferm. USP. 2015;49(1):618.

22. Mendonça LBA, Lima FET, Oliveira SKP. Acidente vascular encefálico como complicação da hipertensão arterial: quais são os fatores intervenientes? Esc Anna Nery. 2012;16(2):340-6.

23. Rangel ESS, Belasco AGS, Diccini S. Qualidade de vida de pacientes com acidente vascular cerebral em reabilitação. Acta Paul Enferm. 2013;26(2):205-12.

24. Barbosa AC, Medeiros LS, Duarte N, Meneses C. Factores preditivos de depressão pós-acidente vascular cerebral: estudo retrospectivo numa unidade de reabilitação. Acta Med Port. 2011;24(Suppl 2):17580 .

25. Dourado CC, Engler TMNM, Oliveira SB. Disfunção intestinal em pacientes com lesão cerebral decorrente de acidente vascular cerebral e traumatismo cranioencefálico: estudo retrospectivo de uma série de casos. Texto Contexto Enferm.2012;21(4):905-11.

26. Rodrigues RAP, Marques S, Kusumota L, Santos EB, Fhon JRS, Fabrício-Wehbe, SCC. Transição do cuidado com o idoso após acidente vascular cerebral do hospital para casa. Rev. Latino-Am. Enferm. 2013;21(n. esp):216-24.

27. Cucolo DF, Perroca MG. Monitorando indicadores de desempenho relacionados ao tempo de assistência da equipe de enfermagem. Rev. Esc. Enferm. USP. 2010;44(2):497-503. 